\title{
Robust Stability Analysis for Barrier-Based Equation-free Multi-linear Model Predictive Control
}

DOI:

10.1016/j.cherd.2018.11.012

\section{Document Version}

Accepted author manuscript

Link to publication record in Manchester Research Explorer

\section{Citation for published version (APA):}

Petsagkourakis, P., Heath, W., \& Theodoropoulos, C. (2019). Robust Stability Analysis for Barrier-Based Equationfree Multi-linear Model Predictive Control. Chemical Engineering Research \& Design, 144, 237-246.

https://doi.org/10.1016/j.cherd.2018.11.012

\section{Published in:}

Chemical Engineering Research \& Design

\section{Citing this paper}

Please note that where the full-text provided on Manchester Research Explorer is the Author Accepted Manuscript or Proof version this may differ from the final Published version. If citing, it is advised that you check and use the publisher's definitive version.

\section{General rights}

Copyright and moral rights for the publications made accessible in the Research Explorer are retained by the authors and/or other copyright owners and it is a condition of accessing publications that users recognise and abide by the legal requirements associated with these rights.

\section{Takedown policy}

If you believe that this document breaches copyright please refer to the University of Manchester's Takedown Procedures [http://man.ac.uk/04Y6Bo] or contact uml.scholarlycommunications@manchester.ac.uk providing relevant details, so we can investigate your claim.

\section{OPEN ACCESS}




\title{
Robust Stability Analysis for Barrier-Based Equation-free Multi-linear Model Predictive Control
}

\author{
Panagiotis Petsagkourakis ${ }^{\mathrm{a}}$, William P. Heath ${ }^{\mathrm{b}}$ and Constantinos Theodoropoulos $^{\mathrm{a} *}$

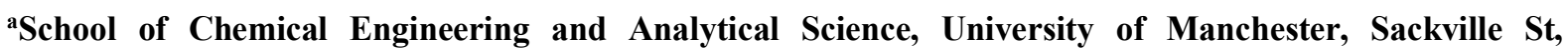 \\ Manchester M13 9PL, UK

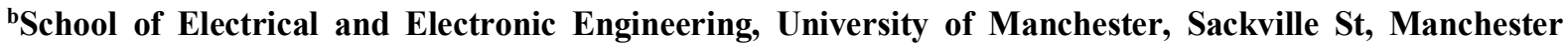 \\ M13 9PL, UK
}

*Corresponding author. Tel.: +44 1613064386. E-mail address: k.theodoropoulos@manchester.ac.uk (C. Theodoropoulos).

\begin{abstract}
On-line optimization strategies such as model predictive control (MPC) have been widely used to compute control actions for a range of complex industrial systems. Barrier based MPC has recently been introduced, bringing together theory and algorithms for analysing the stability of linear models, however such models may not describe complex systems dynamics adequately. Multi-model linear MPC configurations can be used as a more reliable solution as piecewise affine (PWA) models can describe the underlying nonlinear dynamics more accurately. Additionally, model order reduction can be applied to large-scale distributed systems, to reduce their dimensionality, jeopardising however their closed-loop stability. As a result, there is a clear need for an input to output stability analysis for closed loop systems under unstructured uncertainty when multi-model barrier MPC is utilized. In this work, we combine equation-free model reduction with integral quadratic constraints (IQCs) for the stability analysis of large-scale closed-loop systems under unstructured uncertainties, including model approximation errors and nonlinearities, including MPC. An illustrative example is used to elucidate the proposed methodology.
\end{abstract}

Keywords: integral quadratic constraints; dissipative systems; model reduction; equation-free; piece-wise affine.

\section{Introduction}

Model predictive control (MPC) has found widespread application in the process industries (Mayne, 2014). Nevertheless, the increased use of high-fidelity models produces complex large-scale systems requiring, often impractically, significant computational resources. Therefore the use of reduced order models (ROM) (Bonis et al., 2014a; Sopasakis et al., 2013) together with the so-called fast MPC (Bock et al., 2007; Jerez et al., 2014; Stella et al., 2017; Wang and Boyd, 2010) or explicit MPC (Bemporad et al., 2002) has gained a significant attention.

Robust stability is a major issue for ROM-based MPC due to the presence of model error. For linear systems, a framework for input-state stability has been recently developed though the inclusion of a re-centered barrier (Adrian G Wills and Heath, 2004) and further extended to relaxed Barrier MPC (Feller and Ebenbauer, 2016, 2015a, 2015b). The latter approach brings together linear MPC theory and algorithms enabling to construct stable controllers with real-time computation guarantees. The main disadvantage of this approach is the 
requirement for relaxed input and state system constraints. Moreover, it is still not trivial to guarantee robust stability when unstructured uncertainty is present. In (Heath et al., 2006) the input-output stability of linear MPC under structured and unstructured uncertainties is considered, extending the results of (Heath et al., 2005), which provide nominal stability limits for output feedback MPC. The advantage of the input-output analysis is that global stability can be guaranteed. One of the main tools used in input-output stability are the integral quadratic constraints (IQCs) introduced by (Yakubovich, 1967) and popularised by (Megretski and Rantzer, 1997). These have been used to perform robust analysis of dynamic and control systems by describing the input-output behaviour of nonlinear uncertainties. Through the use of IQCs, stability conditions in the frequency domain can be transformed to a set of linear matrix inequalities (LMIs) using the KYP lemma (Rantzer, 2012). Recently, stability theorems have been introduced in time domain using dissipation theory (Lozano et al., 2000) and timedomain IQCs (Pfifer and Seiler, 2015) using the Parserval's theorem (Lozano et al., 2000). However, dissipation theory requires finite time constraints and in (Pfifer and Seiler, 2016) hard IQCs are utilized after transforming the soft constraints into hard constraints using $J$-factorization. A common uncertainty source in process control is the model error originating from simplifications that may be applied on a model, such as linearization or model reduction, which depends on the current states and can be described as unstructured error. Model reduction plays an important role in MPC, as systems with a large number of variables can be an obstacle for the implementation of optimizing controllers. A number of model reduction techniques for control applications have been reviewed in (Theodoropoulos, 2011). In addition, recent studies have explored model reduction through time-domain partitioning and combined it with MPC (Narasingam and Kwon, 2017). In this work we apply the equation-free framework (Theodoropoulos et al., 2000) to compute a reduced system from input/output information only. This model reduction technique requires the system to be dissipative, i.e. the existence of a separation between its (relatively few) slow modes, which determine its dynamic performance and its fast modes, which are enslaved to the dominant slow modes. Dissipative PDEs modelling reaction and diffusion phenomena are characterized by such property.

In the work of (Hovland et al., 2008) the stability framework of (Løvaas et al., 2010) has been exploited in order to provide a design methodology when model reduction (using balanced truncation) is applied in LTI models. IQCs have been utilized to take into account unstructured uncertainties for stability analysis. Recently, we developed a stability framework utilizing some novel IQCs for the case of multi-model MPC and PWA systems under unstructured uncertainty (Petsagkourakis et al., 2018). In this work we extend our framework to incorporate barrier based MPC controllers for multi-model-based schemes after equation-free model reduction has been applied. The structure of the controller has been explored such that convex and/or soft constraints can be incorporated in the analysis. The theoretical and algorithmic advantages of the use of barriers in MPC have been explored for linear models in the literature (Feller and Ebenbauer, 2015a; A. G. Wills and Heath, 2004).

Here, time domain hard IQCs are constructed in order to include the effect of model approximation in stability analysis. In this way the controller can employ nonlinear convex constraints and have satisfactory performance with the multi-model scheme. As a result, in this work new IQCs are developed together the stability theorems extending the work of (Petsagkourakis et al., 2018). The proposed methodology is particularly useful to analyse PWA with unstructured uncertainty, if there exists an upper bound of the model mismatch. The paper is arranged as follows: Relevant notation is given in section 2. In section 3 the general methodology is presented including the formulation of the problem, a brief introduction of equation-free model reduction, barrier based 
MPC as well as background on the theory of time-domain IQCs. Section 4 includes the main results of the paper and an illustrative chemical engineering case study. Finally conclusions and future work directions are given in section 5 .

\section{Notations}

Let $\mathbb{Z}$ and $\mathbb{Z}_{+}$be the set of integer numbers and positive integer numbers including 0 , respectively. $l_{2}^{m}$ is the Hilbert space of all square integrable and Lebesque measurable functions $f: \mathbb{Z}_{+} \rightarrow \mathbb{R}^{m}$. Let $l^{m}$ be the space of all real valued sequences (this is the extended space of $l_{2}^{m}$ ), so the truncation of the function $f=f(t)$ at $T$ is defined as:

$$
f_{T}(t)= \begin{cases}f(t), \forall t \leq T \\ 0, \quad \forall t>T\end{cases}
$$

The function $f$ belongs to the extended space $l^{m}$ if $f_{T} \in l_{2}^{m}$ for all $T>0 . \mathbb{R H}_{\infty}$ stands for the set of rational transfer function matrices without poles outside the unit circle. For a matrix $A, A^{*}$ is its complex conjugate transpose. Additionally, $G^{*}$ is the $l_{2}$-adjoint operator of $G$. $\langle f, g\rangle$ is the inner product defined as $\sum_{k=0}^{\infty} f_{k}^{T} g_{k}=$ $\frac{1}{\pi} \int_{-\pi}^{\pi} \hat{f}\left(e^{i \omega}\right) \hat{g}\left(e^{i \omega}\right) d \omega$ and the $l_{2}$-norm $\|f\|_{2}$ is defined as $\sqrt{\langle f, g\rangle}$, while $\|f\|_{1}$ is $\sum_{k=0}^{\infty}\left|f_{k}\right|$. Here $\hat{f}$ denotes the discrete Fourier transform of $f$. Furthermore, $G_{j}$ is a piece-wise affine (PWA) model with $j$ the index for each model. Moreover, the size of a signal $x$ is indicated as $n_{x}$.

\section{Methodology}

\subsection{Problem Formulation}

In this work, the control of a dissipative infinite dimensional system is considered where a black-box simulator is available to simulate the physical system. An equation-free (EF) methodology is applied in order to reduce the size of the system using only an available black-box simulator. This scheme combines with a clustering sampling approach (Petsagkourakis et al., 2018) produces a model pool of multiple reduced order sub-models (see section 3.2) resulting in a reduced piecewise affine (rPWA) model under unstructured uncertainty, which efficiently approximates the highly nonlinear infinite dimensional system:

$$
\begin{gathered}
x(k+1)=A_{j} x(k)+B_{j} u(k)+f_{j} \\
y(k)=C_{j} x(k) \\
j \in \mathcal{M}
\end{gathered}
$$

Here $x \in \mathbb{R}^{n_{x}}$ corresponds to the vector of the reduced states, $u \in \mathbb{R}^{n_{u}}$ are the inputs (manipulated variables) of the physical system and $y \in \mathbb{R}^{n_{y}}$ is the vector of the output (control) variables. Furthermore, every $A_{j}$ is considered to be Hurwitz and $\mathcal{M}$ is the set that contains all the reduced order sub-models. Model-based control of such systems may be challenging, the online optimization may be computationally expensive either because of the size of the constraints or the combinatorial nature of the PWA-based MPC, despite the reduced size of the states. Hence a relaxed barrier MPC formulation (Feller and Ebenbauer, 2017) is employed using a multi-model scheme (Bonis et al., 2014a; Du et al., 2013). Only input constraints are utilized in our analysis as the aim is to predict the limits of global input-to-output stability. It should be note that the accuracy of the reduced model(s) 
is never perfect and the model error clearly depends on the system's output. In addition, the stability of the relaxed barrier MPC formulation needs to be proven. As a result in this work a global stability analysis for relaxed barrier equation-free (EF) based multi-model MPC (EF-rMPC) under unstructured uncertainty is performed.

\subsection{Equation-free Model Reduction}

In this section equation-free model reduction is introduced, which we utilize to construct a reduced order model pool. It has been widely implemented for bifurcation analysis (Theodoropoulos et al., 2000), optimization (Bonis and Theodoropoulos, 2012) and control (Armaou et al., 2005; Bonis et al., 2012) applications. The basis of this technique is the natural partition of a dissipative system's dynamics into slow and fast modes. This behaviour is present in parabolic PDEs (Temam, 1997), where the number of the dominant slow modes is significantly smaller than the fast modes. First, we introduce the form of the main physical system as:

$$
\begin{aligned}
& \frac{\partial x_{p}}{\partial t}=\mathcal{A}\left(\frac{\partial x_{p}}{\partial z}, \frac{\partial^{2} x_{p}}{\partial z^{2}}, \ldots, \frac{\partial^{n} x_{p}}{\partial z^{n}}\right)+B(z) u+F\left(x_{p}\right) \\
& y=\int_{\Omega} c(z) x_{p} d z
\end{aligned}
$$

where $x_{p}: \Omega \times \mathbb{R}_{+} \rightarrow \mathbb{R}^{n_{x_{p}}}$ are the system states, $z \in \Omega$ the spatial dimension, $\Omega$ is defined as an open bounded set of $\mathbb{R}^{n_{z}}$ and $n_{x_{p}}, n_{z}$ the number of states and the size of the spatial dimensions respectively. Additionally, the physical system is considered to have mixed-type boundary conditions. It is common to have an available stable black-box simulator (e.g. a commercial CFD simulator such as COMSOL or FLUENT) that can efficiently simulate such physical systems for a specific time horizon, given a set of initial conditions, and parameters $(u)$. These simulators produce an approximation of $x_{p}(z, t)$ for a finite spatial domain and a finite time domain:

$$
\left[x_{p}(1,(k+1) \tau)^{T}, \ldots, x_{p}\left(z_{1},(k+1) \tau\right)^{T}, \ldots\right]^{T} \approx x(k+1)=G_{\tau}(x(k), u(k))
$$

where $x(k) \in \mathbb{R}^{n \cdot n_{x_{p}}}$ is a finite state approximation of $x_{p}$ at the time instant $k \tau, \tau$ being the sampling time. Such simulators only provide input/output information to the user; as a result an equation-free methodology is suitable in order to efficiently reduce the size of the physical system.

The dissipativity property of such infinite-dimensional systems, with the concomitant separation of scales, allows us to find a finite dimensional expression that can approximate the system's behaviour as a result of the spectral gap in the eigen-spectrum (Temam, 1997; Zelik, 2014). This separation of scales is depicted by the eignespectrum in Fig. 1: 


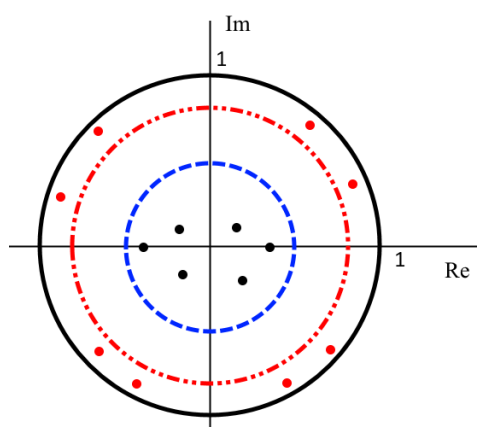

Figure 1: Separation of scales in eigen-spectrum

The eigenvalues close to the unit circle correspond to the slow (and/or unstable) dominant modes and those close to zero correspond to fast modes that are enslaved by the slow ones. The model reduction is based on the fact that there are only few $m$ eigenvalues close to the unit circle. Now, let $\mathcal{P}$ denote the maximal invariant subspace that corresponds to the dominant modes and $\mathcal{Q}$ its orthogonal complement, so that:

$$
\mathcal{P} \oplus \mathcal{Q}=\mathbb{R}^{n \cdot n_{x_{p}}}
$$

An approximation, $\hat{Z}$, of the orthonormal basis $Z \in \mathbb{R}^{n \cdot n_{x_{p} \times m}}$ for the subspace $\mathcal{P}$ can be computed through subspace or Arnoldi iterations. These methods are considered as matrix-free and the use of the full Jacobian is not required. The vector of system states, $x$, can be replaced with its low-dimensional projection, $x_{r}$, onto $\mathcal{P}: x_{r}=\hat{Z}^{T} x$. the full states $x$ can be now decomposed as:

$$
x=P x+Q x
$$

If the fast modes are disregarded $Q x \approx 0$ and the system can be adequately approximated with only $m$ modes

$$
x \approx P x=\hat{Z} x_{r}
$$

Ideally, the orthonormal basis would be updated at every sampling time; however that would be computationally expensive compromising the overall computational efficiency of the model reduction scheme. In this work, a set of sub-models is employed, in which every sub-model has a different pre-computed basis.

The set of sub-models $\mathcal{M}$ is constructed off-line and this procedure starts with a collection of temporal trajectories using an available simulator. Subsequently, principle component analysis (PCA) is employed and the resulted modes are clustered. In this paper a $k$-means (Hastie et al., 2009) algorithm is implemented and more details can be found in (Petsagkourakis et al., 2018). The optimal construction of reduced order models is out of the scope of this work; however, systematic approaches such the one proposed in (P. Tan et al., 2007) can be applied where global optimum search is utilized to compute the optimum clusters. The centroids computed by $k$-means are used to compute the reduced bases as well as all the sub-models employing EF model reduction. The corresponding reduced Jacobians $A_{r_{j}}$ can efficiently be computed using matrix-free Arnoldi iterations, where only matrix-vector products are required:

$$
\nabla_{x} G_{\tau} \widehat{Z}_{j}^{i} \approx \frac{G_{\tau}\left(x+\epsilon \widehat{\mathbf{Z}}_{j}^{i}\right)-G_{\tau}\left(x-\epsilon \widehat{\mathbf{Z}}_{j}^{i}\right)}{2 \epsilon}
$$

Here $j$ corresponds to the $j^{t h} \in \mathcal{M}$ centroid, $\hat{Z}_{j}^{i} \in \mathbb{R}^{n \cdot n_{x_{p}}}$ is the $i^{t h}$ column of $\hat{Z}_{j}$ and $\epsilon$ a positive small number. The simulator employs these perturbations by running the dynamic model for a time horizon $\tau$, for given initial 
conditions and parameters $u$. Multiplying $\hat{Z}^{j T}$ with $\nabla_{x} G_{\tau} \hat{Z}^{j}$ produces the desired reduced Jacobian ( Shroff and Keller, 1993; Bonis et al., 2012) .

$$
A_{r_{j}}=\hat{\mathrm{Z}}_{j}^{T} \nabla_{x} G_{\tau} \hat{Z}_{j}
$$

The resulted orthonormal basis $\hat{Z}_{j}$ and reduced Jacobian $\left(A_{r_{j}}\right)$ are then used to construct each sub-model in the form of Eq. 1.

Each model is computed at the centroid of each cluster; hence the model selection at each time can be performed either with respect to the distance between the current sampling point and the centroids or with respect to one of the polyhedrals (containing the sampling point) produced by Voronoi diagrams, or by multi-category linear separation methods (Breschi et al., 2016) for the cluster centroids.

Until this point the assumption that Eq. 6 holds has been made. Nevertheless, this may not always be valid, especially when an off-line construction of sub-models has been performed using collected trajectories. As a result, Eq. 6 can be re-written as $x=\hat{Z} x_{r}+\epsilon_{r}$, with $\epsilon_{r}$ being the error from the model reduction. The estimation of this error is not always easy as the full system is highly nonlinear and unavailable. Additionally, there is error originating from the linearization, which should also be considered. Nevertheless, this error is usually much smaller as there are enough models to approximate different regions. Hence, in the general case, for each model, $j$, this error can be seen as:

$$
\left\|G_{\tau}(x(k), u(k))-\hat{Z}_{j}\left(A_{r_{j}} x_{r}(k)+B_{r_{j}} u(k)+f_{r_{j}}\right)\right\|_{2} \leq L\left\|x(k)-x_{r}(k)\right\|_{2}
$$

assuming that the total model error is norm-bounded. This error is unstructured and depends on the output of the real system. However, the upper bound of this error is not trivial to be estimated, unless additional assumptions are made for the dynamic system (Rewienski and White, 2003). In our work, an input-to-output stability analysis is proposed that accounts for this unstructured error (model error) for any given error bound, and any other nonlinear component that admits an integral quadratic constraint (IQC).

\subsection{Dissipation Inequality}

The equation-free model reduction that was described in section 3.2 is based on dissipativity, a property that the physical systems (such as those that are described by parabolic PDEs) may have, resulting in a separation of scales of the system's eigen-spectrum, allowing us to describe an infinite dimensional system with a finitereduced order one. In this section, stability analysis is described in terms of dissipativity, expressed through the dissipation inequality (Eq. 11) in order to analyse the stability of the closed-loop system depicted in Fig. 2. Hence, dissipativity has been exploited twice, but for different purposes.

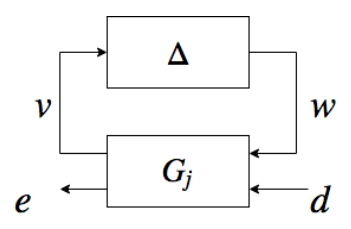

Figure 2: Closed-loop system. $G_{j}$ represents all the PWA and linear parts, and $\Delta$ all the uncertainties/nonlinearities. 
The induced $l_{2}$ norm is given by Eq. 10 from the external input $d$ to $e$ :

$$
\left\|\left(G_{j}, \Delta\right)\right\|=\sup _{d \in l_{2}} \frac{\|e\|}{\|d\|}
$$

Where $G_{j}$ represents all the PWA and linear parts and $\Delta$ is the augmented uncertainty including all unstructured uncertainties and nonlinearities, namely model error and rMPC, in diagonal form (Jönsson and Rantzer, 2000).

Definition 1(Lozano et al., 2000)

A system is said to be dissipative if there exists a "storage function" $V(x) \geq 0$ such that the dissipation inequality holds:

$$
V(x)-V\left(x_{0}\right) \leq \sum_{k=0}^{T} s(d(k), e(k))
$$

along all possible trajectories of the system starting at $x_{0}$ and $T>0$.

From the above definition it is clear that the selection of a storage function is important for the analysis of the closed-loop system, as different type of storage functions may provide different degrees of conservatism. In this work two storage functions are employed: the common storage function and the piecewise quadratic (PWQ) storage function. For more details about the selection of storage function the reader should be referred to (Rantzer and Johansson, 2000). Briefly, the common storage function is parameterized with a quadratic term, $V=x^{T} P x$, and the PWQ is parametrized as $V=x^{T} P_{j} x$. The conservatism of the analysis, in the case of PWQ drops as different $P_{j}$ can be found for different sub-models. Nevertheless, the use of PWQ storage function significantly increases the computational load, and may produce intractable computational problems. Therefore, it is important to take advantage of all the excess positivity that the nonlinearity may be able to provide, in order to be less conservative with the use of common storage functions.

The robustness of the stability of the closed-loop system is analysed using an extended system, $G_{s}$, with states $x^{s}:=\left[\begin{array}{l}x_{r} \\ \psi\end{array}\right]$. Without loss of generality $U_{s s}$ (as an external signal) is assumed to be 0 in order to keep the notations and equations simple. The extended system is shown in Figure 3, where $\psi$ corresponds to the states of $\Psi$, the filter of the time-domain IQCs. In our work all the IQC multipliers are considered to be static, hence the identity matrix can be employed as a filter. Additionally, the model that describes the main physical system is assumed to be PWA, therefore the extended system is PWA as well:

$$
\begin{gathered}
x^{s}(k+1)=A_{j}^{s} x^{s}(k)+B_{j}^{s_{1}} w(k)+B_{j}^{s_{2}} d(k) \\
z(k)=C_{j}^{s_{1}} x^{s}(k)+D_{j}^{s_{11}} w(k)+D_{j}^{s_{12}} d(k) \\
e(k)=C_{j}^{s_{2}} x^{s}(k)+D_{j}^{s_{21}} w(k)+D_{j}^{s_{22}} d(k)
\end{gathered}
$$

The structure of the matrices in the extended state space depends on the underlying problem and in order to avoid confusion, a particular structure is not given. 


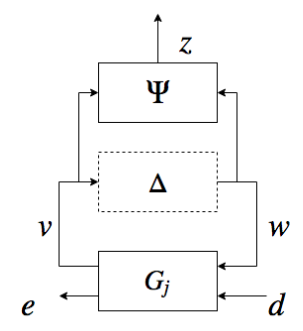

Figure 3: Extended System

In the next sections the concept of relaxed barrier-based IQCs is introduced.

\subsection{Relaxed Re-centered Barrier Equation Free Based Multi-model MPC}

The idea behind Barrier based MPC is the construction of an unconstrained problem that is strongly convex for the case of linear models and linear constraints. In this section we briefly discuss the formulation of such problems in the case of multi-model MPC. The barrier MPC has been enhanced by the use of a re-centered barrier (Adrian G Wills and Heath, 2004) (or the weight re-centered barrier (Feller and Ebenbauer, 2015a)) with the resulting optimization problem being strongly convex and unconstrained. The barrier function used in this work is the re-centered formulation as in (Wills and Heath, 20S04) and a quadratic penalty is applied when the constraints are relaxed (Feller and Ebenbauer, 2015a):

Let $C$ be an open non-empty convex set which contains the origin. Let $\bar{B}: C \subset \mathbb{R}^{n}$ be a self-concordant barrier function over $C$. Define a function: $C \subset \mathbb{R}^{n}$ as

$$
B(q)=\bar{B}(q)-\bar{B}\left(q_{0}\right)-\nabla_{U} \bar{B}\left(q_{0}\right)\left(q-q_{0}\right)
$$

Then, $B$ is called gradient recentred self-concordant barrier function over $C$.

Self-concordant barrier $\bar{B}$ for the case of input constraints can be defined as

$$
\bar{B}(U)=-\sum_{i=1}^{N_{\text {constr }}} \ln \left(b_{i}-F_{i}(U)\right)
$$

with $N_{\text {constr }}$ being the number of input constraints and $b_{i}-F_{i}(U) \geq 0$ the $i^{t h}$ constraint. In this work, such constraints are also considered extending the methodology of (Petsagkourakis et al., 2018) so that general convex constraints for the input variables can be imposed, besides linear constraints.

The barrier-based unconstrained optimization problem may be compromised by the infeasibility from the use of hard constraints as in Eq. 14. This is more common when state (or possibly) output constraints are present. Nevertheless, this issue will also arise for nonlinear input constraints. For this reason, a relaxed formulation of the barrier function was introduced in (Feller and Ebenbauer, 2015a). Hence the barrier function in Eq. 13 can be substituted by the re-centered barrier, $B_{r}$, given by the following twice continuously differentiable function:

$$
B_{r}(U)=\left\{\begin{array}{c}
\bar{B}(U)-\bar{B}\left(U_{s s}\right)-\nabla_{U} \bar{B}\left(U_{s s}\right)\left(U-U_{s s}\right), \quad b_{i}-G_{i}(U) \geq \delta_{i} \\
\left(\left[\frac{b_{i}-G_{i}(U)-2 \delta_{i}}{\delta_{i}}\right]^{2}-1\right)-\ln \left(\delta_{i}\right)-\bar{B}\left(U_{s S}\right)-\nabla_{U} \bar{B}\left(U_{s s}\right)\left(U-U_{s S}\right), \quad \text { otherwise }
\end{array}\right.
$$


where $\delta_{i}$ is the relaxation parameter. $B_{r}$ is a strictly convex function (Feller and Ebenbauer, 2015a), and $B_{r} \rightarrow B$ for $\delta_{i} \rightarrow 0$. In this work, we show that the stability regions are unchanged for both the regular and the relaxed barrier formulation.

Let us now consider the barrier-based optimization problem:

$$
\begin{gathered}
U^{*}=\underset{U}{\operatorname{argmin}} \frac{1}{2}\left[\sum_{i=2}^{M_{\text {out }}}\left(y(i)-y_{r e f}\right) Q\left(y(i)-y_{r e f}\right)+\sum_{i=1}^{M_{\text {in }}}\left(U(i)-U_{s s}\right) R\left(U(i)-U_{s s}\right)\right]+\mu B(U)(16) \\
\text { s.t } x_{r}(i+1)=A_{r_{j}} x_{r}(i)+B_{r_{j}} u(i)+f_{r_{j}} \\
y_{i}=C_{r_{j}} x_{r_{i}}
\end{gathered}
$$

where $j$ is the selected sub-model at the current time. The constraints that are considered in this problem are in the form of $b_{i}-F_{i}(U) \geq 0$ and $\mu$ is a positive relaxation parameter (Boyd and Vandenberghe, 2004). This problem can be easily transformed to an unconstrained problem, simply by substituting the equality constraints to the objective function.

$$
\phi\left(x_{r}\right)=U^{*}=\underset{U}{\operatorname{argmin}} \frac{1}{2} U^{T} H_{j} U-\left[\begin{array}{c}
x_{r} \\
U_{s s} \\
y_{r e f}
\end{array}\right]^{T} F_{j}^{T} U+f_{r_{j}} L_{3}^{T} U+\mu B_{r}(U)
$$

The last equation is a convex unconstrained problem that can be solved fast by an appropriate convex programming algorithm (Patrinos and Bemporad, 2013). The form of $H_{j}$ and $F_{j}=\left[L_{0}, L_{1}, L_{2}\right]$ can been found in (Heath et al., 2006). The barrier in this case is re-centered to the input variable at the steady-state that corresponds to the set-point. These values can be found by solving the following optimization problem:

$$
U_{s s}=\arg \min _{u}\left\|y_{r e f}-C_{r_{j}}\left(I-A_{r_{j}}\right)^{-1}\left(f_{r_{j}}-B_{r_{j}} u\right)\right\|_{2}
$$

Even though the barrier based MPC is a nonlinear optimization problem, IQCs can be found that describe the input-output behaviour of the nonlinear map.

\subsection{Integral Quadratic Constraints}

This section briefly introduces IQCs and more information can be found in (Megretski and Rantzer, 1997). Integral quadratic constraints (IQCs) provide a useful characterization of a given operator on a Hilbert space and they are defined in terms of self-adjoint operators. Consider the following nonlinear/uncertain system

$$
w=\Delta(\mathbf{v})
$$

with $\Delta: \mathbb{R}^{m} \rightarrow \mathbb{R}^{m}$ the uncertainty or nonlinearity that affects the system, $v \in l^{m}\left(\mathbb{Z}_{+}\right)$and $w \in l^{m}\left(\mathbb{Z}_{+}\right)$its input and output, respectively. If $\Delta$ takes the form of eq.19 then for all $v \in l^{m}\left(\mathbb{Z}_{+}\right)$the following IQC is said to be satisfied if

$$
\begin{gathered}
\left\langle\left[\begin{array}{l}
v \\
w
\end{array}\right], \Pi\left[\begin{array}{l}
v \\
w
\end{array}\right]\right\rangle= \\
\int_{-\pi}^{\pi}\left[\begin{array}{c}
\hat{v}\left(e^{j \omega}\right) \\
\widehat{w}\left(e^{j \omega}\right)
\end{array}\right], \Pi\left(e^{j \omega}\right)\left[\begin{array}{c}
\hat{v}\left(e^{j \omega}\right) \\
\widehat{w}\left(e^{j \omega}\right)
\end{array}\right] d \omega \geq 0 \\
\forall v, w \in l^{m}\left(\mathbb{Z}_{+}\right)
\end{gathered}
$$


Where, $\Pi$, is a bounded and self-adjoint operator that defines the IQC, $\hat{v}$ and $\hat{w}$ are the corresponding Fourier transformations. $\Pi$ can be factorized, $\Pi=\Psi^{*} M \Psi, M$ being the IQC multiplier and $\Psi$ a filter. The notation that is usually used is $\Delta \in \operatorname{IQC}(\Pi)$. This formulation is useful when the physical system is linear; however in this work, the resulting system is PWA under unstructured uncertainty and a different strategy should be implemented. In previous work (Petsagkourakis et al., 2018) we have introduced a piecewise IQC to fit to the needs of PWA models as in Eq. 21

$$
\sum_{k=0}^{T} r^{T}(k) M_{j(k)} r(k) \geq 0
$$

where $j(k)$ corresponds to the sub-model $j$ at time $k, M_{j(k)}$ to the multiplier of the piece-wise IQC, and $r:=\Psi\left(e^{j \omega}\right)\left[\begin{array}{l}v \\ w\end{array}\right]$. In fact, the model does not change with respect to time but with respect to output, hence it is $j(y(k))$ but will be denoted as $j(k)$. In this work, we extend the methodology proposed in (Petsagkourakis et al., 2018) in order to include the relaxed barrier based MPC, as well as the equation-free model reduction. In the next chapter, the IQCs for the case of the relaxed Barrier are developed followed by the proposed stability analysis.

\section{Results and Discussion}

\subsection{Integral Quadratic Constraints for Relaxed Re-centered Barrier Model Predictive Control}

In this section the properties of relaxed re-centered barrier are employed to formulate the IQCs. The corresponding strictly convex unconstrained optimization problem is given in Eq. 16 with the KKT conditions in Eq. 22:

$$
H_{j} U-F_{j}\left[\begin{array}{c}
x_{r}(k) \\
U_{s s} \\
y_{r e f}
\end{array}\right]+L_{3} f_{r_{j}} U+\mu \nabla_{U} B_{r}(U)=0
$$

The relaxed barrier function is convex. As a result, its derivative with respect to the input variable is monotonically increasing for general nonlinear convex constraints, hence the following holds:

$$
\left(U_{2}-U_{1}\right)\left(\nabla_{U} B_{r}\left(U_{2}\right)-\nabla_{U} B_{r}\left(U_{1}\right)\right) \geq 0
$$

Because the barrier function is re-centered $\nabla_{U} B_{r}\left(U_{s s}\right)=0$, therefore $\left(U-U_{s s}\right)\left(\nabla_{U} B_{r}(U) \geq 0\right.$. As a result, Lemma 1 follows immediately.

\section{Lemma 1}

The nonlinearity given by Eq. 16 admits the following $I Q C \forall p \in l^{n_{p}}$ :

$$
\sum_{k=0}^{T}\left[\begin{array}{l}
p(k) \\
q(k)
\end{array}\right]^{T}\left[\begin{array}{cc}
0 & I \\
I & -2 H_{j(k)}
\end{array}\right]\left[\begin{array}{l}
p(k) \\
q(k)
\end{array}\right] \geq 0
$$

with $q=U-U_{s s}$ and $p=x_{r}-x_{s s}$ then

Proof:

The KKT conditions can now be written as 


$$
\left(U-U_{S S}\right)^{T} H_{j} U-\left(U-U_{s S}\right)^{T} F_{j}\left[\begin{array}{c}
x_{r}(k) \\
U_{s s} \\
y_{r e f}
\end{array}\right]+\left(U-U_{s S}\right) L_{3} f_{r_{j}} U=-\left(U-U_{s S}\right)\left(\nabla_{U} B_{r}(U) \leq 0\right.
$$

Inequality (25) holds for the case where a relaxed re-centered barrier (Eq. 16) is introduced, as the monotonicity is preserved. Therefore

$$
\left(U-U_{s s}\right)^{T} H_{j}\left(U-U_{s s}\right)-\left(U-U_{s s}\right)^{T} L_{0}\left[x_{r}(k)-x_{s s}\right] \leq 0
$$

with $x_{s s}$ corresponding to the steady-state solution of Eq. 1 coupled with Eq. 18 . The summation from $\mathrm{k}=0$ to $T$ transforms inequality (26) to an IQC

$$
\sum_{k=0}^{T}\left[\begin{array}{l}
p(k) \\
q(k)
\end{array}\right]^{T}\left[\begin{array}{cc}
0 & I \\
I & -2 H_{j(k)}
\end{array}\right]\left[\begin{array}{l}
p(k) \\
q(k)
\end{array}\right] \geq 0
$$

In inequality (27) The static multiplier $\Pi=\left[\begin{array}{cc}0 & I \\ I & -2 H_{j(k)}\end{array}\right]$ can be factorized by defining $\Psi=\left[\begin{array}{ll}I & 0 \\ 0 & I\end{array}\right]$ and $M=\left[\begin{array}{cc}0 & I \\ I & -2 H_{j(k)}\end{array}\right]$. Even though this result is important in order to compute global stability conditions for barrier-based multi model MPC (see section 4.3), the outcome is expected to be conservative as there is no efficient parametrization on the IQCs. To tackle this problem a conical combination can be applied using the results of Lemma 1.

\section{Lemma 2}

The nonlinearity given by Eq. 16 admits the following $I Q C \forall p \in l^{n_{p}}$ :

$$
\sum_{k=0}^{T} \lambda_{j(k)}\left[\begin{array}{l}
p(k) \\
q(k)
\end{array}\right]^{T}\left[\begin{array}{cc}
0 & I \\
I & -2 H_{j(k)}
\end{array}\right]\left[\begin{array}{l}
p(k) \\
q(k)
\end{array}\right] \geq 0
$$

Proof:

Inequality (27) holds for each model and each time interval that is applied. For every time interval $k$ that a model $i$ is employed the following holds:

$$
\sum_{k=T_{1}(i)}^{T_{2}(i)}\left[\begin{array}{l}
p(k) \\
q(k)
\end{array}\right]^{T}\left[\begin{array}{cc}
0 & I \\
I & -2 H_{j(k)}
\end{array}\right]\left[\begin{array}{l}
p(k) \\
q(k)
\end{array}\right] \geq 0
$$

Then a conical combination can be applied to give inequality (28). $\square$

Roughly, the parameters $\lambda_{i}$ can exploit an excess positivity in the nonlinear part to redress a deficiency of the positivity in the corresponding linear part.

In the next section, three theorems are given regarding the use of different storage function and IQCs.

\subsection{Common Storage function}

The use of a common storage function may affect the conservatism of the stability analysis. However, the use of (static) multipliers can reduce the conservatism significantly. Hence, the use of a PWQ storage function can be avoided. 


\section{Theorem 1}

Let $G_{i}^{s} \in \mathbb{R} \mathbb{H}_{\infty}^{\left(n_{e}+n_{w}\right) \times\left(n_{w}+n_{d}\right)}$ be a stable system that represents a reduced order model and $\Delta_{i}: l^{n_{v_{i}}} \rightarrow l^{n_{v_{i}}}$ a bounded, causal operator containing the barrier-based multi-model MPC as a nonlinearity and the possible error originated by the model reduction and the linearization. Assume the closed loop is well-posed and every $\Delta_{i}$ satisfies IQCs with multiplier $\Pi_{i}=M_{i}$. The controller (Eq. 16) satisfies IQC with multiplier M ${ }_{1}^{j}$ (see Lemmal). Then $\left\|G_{j}, \Delta\right\|<\gamma$ if there exists a symmetric matrix $P>0$ and nonnegative $\gamma$ and $\lambda=$ $\left[\lambda_{1}, \ldots, \lambda_{i}, \ldots, \lambda_{n_{m}}\right]$ such that $\operatorname{LMI}(\lambda, \gamma, P)$ hold for all $j$ :

$$
\begin{gathered}
L M I(\lambda, \gamma, P):=\left[\begin{array}{ccc}
A_{j}^{S T} P A_{j}^{S}-P & A_{j}^{S T} P B_{j}^{S_{1}} & A_{j}^{S T} P B_{j}^{S_{2}} \\
B_{j}^{S_{1} T} P A_{j}^{S} & B_{j}^{S_{1} T} P B_{j}^{S_{1}} & B_{j}^{S_{1} T} P B_{j}^{S_{2}} \\
B_{j}^{S_{2} T} P A_{j}^{S} & B_{j}^{S_{2} T} P B_{j}^{S_{1}} & B_{j}^{S_{2} T} P B_{j}^{S_{2}}-\gamma^{2} I
\end{array}\right]+ \\
+\left[\begin{array}{c}
C_{j}^{S_{2} T} \\
D_{j}^{S_{21} T} \\
D_{j}^{S_{22} T}
\end{array}\right]\left[\begin{array}{c}
C_{j}^{S_{2} T} \\
D_{j}^{S_{21} T} \\
D_{j}^{S_{22} T}
\end{array}\right]+\left[\begin{array}{c}
C_{j}^{s_{1} T} \\
D_{j}^{S_{11} T} \\
D_{j}^{S_{12} T}
\end{array}\right]\left[\begin{array}{ccc}
\lambda_{1} M_{1}^{j} & \cdots & 0 \\
\vdots & \ddots & \vdots \\
0 & \cdots & \lambda_{N_{m}} M_{N_{m}}
\end{array}\right]\left[\begin{array}{l}
C_{j}^{s_{1} T} \\
D_{j}^{S_{11} T} \\
D_{j}^{S_{12} T}
\end{array}\right]<0
\end{gathered}
$$

Proof:

Using $\gamma^{2}-\delta$ instead of $\gamma^{2}$, the strict inequality turns into a non-strict one. Then, if we multiply inequality (30) with $\left[x^{s T}, w^{T}, d^{T}\right]$ and its transpose from left and right, respectively we get:

$\lambda_{1} r_{1}^{T}(k) M_{1}^{j} r_{1}(k)+\sum_{i=2}^{N_{m}} \lambda_{i} r_{i}^{T}(k) M_{i} r_{i}(k)+V(k+1)-V(k)+e^{T}(k) e(k) \leq\left(\gamma^{2}-\delta\right) d^{T}(k) d(k)$

From the summation of (31) from $k=0$ to $T$ assuming zero initial conditions we have:

$$
\sum_{k=0}^{T} \lambda_{1} r_{1}^{T}(k) M_{1}^{j} r_{1}(k)+\sum_{k=0}^{T} \sum_{i=2}^{N_{m}} \lambda_{i} r_{i}^{T}(k) M_{i} r_{i}(k)+V(T+1)+\sum_{k=0}^{T} e^{T}(k) e(k) \leq\left(\gamma^{2}-\delta\right) \sum_{k=0}^{T} d^{T}(k) d(k)
$$

The storage function, $V$, is positive definite and the IQCs are positive therefore

$$
\sum_{k=0}^{T} e_{k}^{T} e_{k}<\gamma^{2} \sum_{k=0}^{T} d_{k}^{T} d_{k}
$$

Then we have that $\|e\|<\gamma\|d\|$

The above sufficient condition may be conservative. A conic combination can be applied to reduce conservatism. The following theorem uses the scalar static multipliers produced by the conic combination.

\section{Theorem 2}

Let $G_{i}^{s} \in \mathbb{R H}_{\infty}^{\left(n_{e}+n_{w}\right) \times\left(n_{w}+n_{d}\right)} \quad$ be a stable system that represents a reduced order model and $\Delta_{i}: l^{n_{v_{i}}} \rightarrow l^{n_{v_{i}}}$ a bounded, causal operator containing the barrier-based multi-model MPC as a nonlinearity and the possible error originating from the linearization and the model reduction. Assume the closed loop is well-posed and every $\Delta_{i}$ satisfies IQC with multiplier $\Pi_{i}=M_{i}$. The controller (Eq. 16) satisfies multiple IQCs (see Lemma 2). 
Then $\left\|\left(G_{j}, \Delta\right)\right\|<\gamma$ if there exists a matrix $P=P^{T} \geq 0$ and nonnegative $\gamma, \lambda=\left[\lambda_{1}^{j}, \ldots, \lambda_{i}, \ldots, \lambda_{n_{m}}\right]$ such that $\operatorname{LMI}(\lambda, \gamma, P))$ for all $j$ hold

$$
\begin{aligned}
& L M I(\lambda, \gamma, P):=\left[\begin{array}{ccc}
A_{j}^{S T} P A_{j}^{S}-P & A_{j}^{S T} P B_{j}^{S_{1}} & A_{j}^{S T} P B_{j}^{S_{2}} \\
B_{j}^{S_{1} T} P A_{j}^{S} & B_{j}^{S_{1} T} P B_{j}^{S_{1}} & B_{j}^{S_{1} T} P B_{j}^{S_{2}} \\
B_{j}^{S_{2} T} P A_{j}^{S} & B_{j}^{S_{2} T} P B_{j}^{S_{1}} & B_{j}^{S_{2} T} P B_{j}^{S_{2}}-\gamma^{2} I
\end{array}\right]+ \\
& +\left[\begin{array}{l}
C_{j}^{S_{2} T} \\
D_{j}^{S_{21} T} \\
D_{j}^{S_{22} T}
\end{array}\right]\left[\begin{array}{l}
C_{j}^{S_{2} T} \\
D_{j}^{S_{21} T} \\
D_{j}^{S_{22} T}
\end{array}\right]+\left[\begin{array}{c}
C_{j}^{S_{1} T} \\
D_{j}^{S_{11} T} \\
D_{j}^{S_{12} T}
\end{array}\right]\left[\begin{array}{ccc}
\lambda_{1}^{j} M_{1}^{j} & \cdots & 0 \\
\vdots & \ddots & \vdots \\
0 & \cdots & \lambda_{N_{m}} M_{N_{m}}
\end{array}\right]\left[\begin{array}{l}
C_{j}^{S_{1} T} \\
D_{j}^{S_{11} T} \\
D_{j}^{S_{12} T}
\end{array}\right]^{T}<0
\end{aligned}
$$

\section{Proof:}

Using $\gamma^{2}-\delta$ instead of $\gamma^{2}$, the strict inequality turns into a non-strict one. Multiplying inequality (34) with $\left[x^{s T}, w^{T}, d^{T}\right]$ and its transpose from left and right, respectively, we get:

$\lambda_{1} r_{1}^{T}(k) M_{1}^{j} r_{1}(k)+\sum_{i=2}^{N_{m}} \lambda_{i} r_{i}^{T}(k) M_{i} r_{i}(k)+V(k+1)-V(k)+e^{T}(k) e(k) \leq\left(\gamma^{2}-\delta\right) d^{T}(k) d(k)$

The summation of (35) from $k=0$ to $T$ assuming zero initial conditions gives:

$$
\begin{gathered}
\sum_{k=0}^{T} \lambda_{j(k)} r_{1}^{T}(k) M_{1}^{j(k)} r_{1}(k) \geq 0+\sum_{k=0}^{T} \sum_{i=2}^{N_{m}} \lambda_{i} r_{i}^{T}(k) M_{i} r_{i}(k)+V(T+1) \\
+\sum_{k=0}^{T} e^{T}(k) e(k) \leq\left(\gamma^{2}-\delta\right) \sum_{k=0}^{T} d^{T}(k) d(k)
\end{gathered}
$$

The storage function is positive definite and the IQCs are positive therefore

$$
\sum_{k=0}^{T} e^{T}(k) e(k) \leq\left(\gamma^{2}-\delta\right) \sum_{k=0}^{T} d^{T}(k) d(k)
$$

Then we have that $\|e\|<\gamma\|d\|$

Theorem 2 reduces the conservatism of the computation of stability limits, but the use of different models may compromise the stability analysis, hence the use of a single common storage function may not be enough. Therefore, the PWQ variant of the theorem could be applied.

\section{Theorem 3}

Let $G_{i}^{s} \in \mathbb{R H}_{\infty}^{\left(n_{e}+n_{w}\right) \times\left(n_{w}+n_{d}\right)} \quad$ be a stable system that represents a reduced order model and $\Delta_{i}: l^{n_{v_{i}}} \rightarrow l^{n_{v_{i}}}$ a bounded, causal operator containing the barrier-based multi-model MPC as a nonlinearity and the possible error originating from the linearization and the model reduction. Assume the closed loop is well-posed and every $\Delta_{i}$ satisfies IQC with multiplier $\Pi_{i}=M_{i}$. The controller (Eq. 16) satisfies IQC (Lemma2). Then 
$\left\|\left(G_{j}, \Delta\right)\right\|<\gamma$ if there exists a set of matrices $P_{j}=P_{j}{ }^{T} \geq 0$ and nonnegative $\gamma, \lambda=\left[\lambda_{1}^{j}, \ldots, \lambda_{m}, \ldots, \lambda_{n_{m}}\right]$ such that $\operatorname{LMI}\left(\lambda, \gamma, P_{j}\right)$ for all $j$ and $i$ hold

$$
\begin{gathered}
\operatorname{LMI}(\lambda, \gamma, P):=\left[\begin{array}{ccc}
A_{j}^{S T} P_{i} A_{j}^{S}-P_{j} & A_{j}^{S T} P_{i} B_{j}^{S_{1}} & A_{j}^{S T} P_{i} B_{j}^{S_{2}} \\
B_{j}^{S_{1} T} P_{i} A_{j}^{S} & B_{j}^{S_{1} T} P_{i} B_{j}^{S_{1}} & B_{j}^{S_{1} T} P_{i} B_{j}^{S_{2}} \\
B_{j}^{S_{2} T} P_{i} A_{j}^{S} & B_{j}^{S_{2} T} P_{i} B_{j}^{S_{1}} & B_{j}^{S_{2} T} P_{i} B_{j}^{S_{2}}-\gamma^{2} I
\end{array}\right]+ \\
\left.+\left[\begin{array}{c}
C_{j}^{S_{2} T} \\
D_{j}^{S_{21} T} \\
D_{j}^{S_{22} T}
\end{array}\right]\left[\begin{array}{c}
C_{j}^{S_{2} T} \\
D_{j}^{S_{21} T} \\
D_{j}^{S_{22} T}
\end{array}\right]+\left[\begin{array}{c}
C_{j}^{S_{1} T} \\
D_{j}^{S_{11} T} \\
D_{j}^{S_{12} T}
\end{array}\right]\left[\begin{array}{ccc}
\lambda_{1}^{j} M_{1}^{j} & \cdots & 0 \\
\vdots & \ddots & \vdots \\
0 & \cdots & \lambda_{N_{m}} M_{N_{m}}
\end{array}\right]\right]\left[\begin{array}{c}
C_{j}^{S_{1} T} \\
D_{j}^{S_{11} T} \\
D_{j}^{S_{12} T}
\end{array}\right]^{T}<0
\end{gathered}
$$

The proof is similar to the one for Theorem 2 .

\subsection{Case Study}

This framework is applied to the stability analysis of closed-loop tubular reactor system, where an irreversible exothermic reaction takes place. The high-fidelity problem is infinite dimensional and is given by Eq. 39 (Bonis et al., 2012).

$$
\begin{aligned}
& \frac{\partial c}{\partial t}=\frac{1}{P e_{1}} \frac{\partial^{2} c}{\partial z^{2}}-\frac{\partial c}{\partial z}-D a c e^{\frac{\gamma T}{1+T}} \\
& \frac{\partial T}{\partial t}=\frac{1}{P e_{2}} \frac{\partial^{2} T}{\partial z^{2}}-\frac{\partial T}{\partial z}-B D a c e^{\frac{\gamma T}{1+T}}+b\left(T_{w}-T\right)
\end{aligned}
$$

Here $c$ and $T$ are the dimensionless concentration and temperature respectively, while $T_{w}$ is the temperature of the cooling zones. In this case study, the latter temperatures are the manipulated variables of the problem and 8 cooling zones are employed. The system's parameters are $P e_{1}=P e_{2}=7, D a=0.1, B=2, b=1$ and $\gamma_{1}=10$ with Neumann boundary conditions.

The control of such a distributed physical system is a challenging task and model reduction is usually applied (Bonis et al., 2014b) taking advantage of the system's dissipativity (Christofides, 2012). In this work, an equation-free approach is employed according to section 3.2. Only a black-box simulator (in particular MATLAB's PDE solver 'pdepe') is utilized in order to collect a number of trajectories and construct the reduced order set of sub-models with only 21 models of 10 states each using algorithm 1 from (Petsagkourakis et al., 2018). The uncertainty produced by the model reduction together with a possible linearization error is considered as a norm bound uncertainty, $\Delta_{\text {error }}$, as described in section 3.2, with an upper bound $b$. Additionally, in this problem re-centered barrier-based multi-model equation-free MPC is applied in order to build an unconstrained optimization problem with the following nonlinear convex constraint

$$
T_{w_{1}}^{2}+T_{w_{2}}^{2} \leq 0.3
$$

which represents actuator restrictions. This further extends the framework we introduced earlier (Petsagkourakis et al., 2018). The other linear constraints are bounds of the manipulated variables: $-0.1 \leq T_{w_{i}} \leq 0.4$ for $i=$ $1, \ldots, 8$. For the controller 5 and 2 time steps are chosen for the prediction and control horizon respectively and the objective function for the MPC optimization is the same as in section 3.3. It should be noted that in this case 
it is considered that all the states are measurable and there is no need for an observer. However, the analysis can be easily modified to include observers. Stability analysis is carried out, to compute stability boundaries of the closed loop system, and the degrees of freedom are the weight of the objective function $r$ (Eq. 16) and the upper bound of the uncertainty, $b$. This framework is key for the analysis of the system as it can show the limits of the MPC design, since small values for the parameter $r$ can produce a more aggressive controller and destabilize the system. In this application both common and PWQ storage functions are employed. In Figure 4 the results of the stability analysis are shown for the case of a common storage function. The unstable regions are denoted with blue, and the stable regions with cyanic, for the results given by Theorem 1, and yellow the additional stable regions using the conic combination (Theorem 2). As expected in the case of conic combination the conservatism is dropped, albeit slightly. The results using PWQ storage function did not change the result, which means that excessive positiveness has been exploited and there is no need for the use of PWQ. The computations with common storage function need $\sim 4$ seconds, while the PWQ problems need $\sim 10 \mathrm{~min}$.

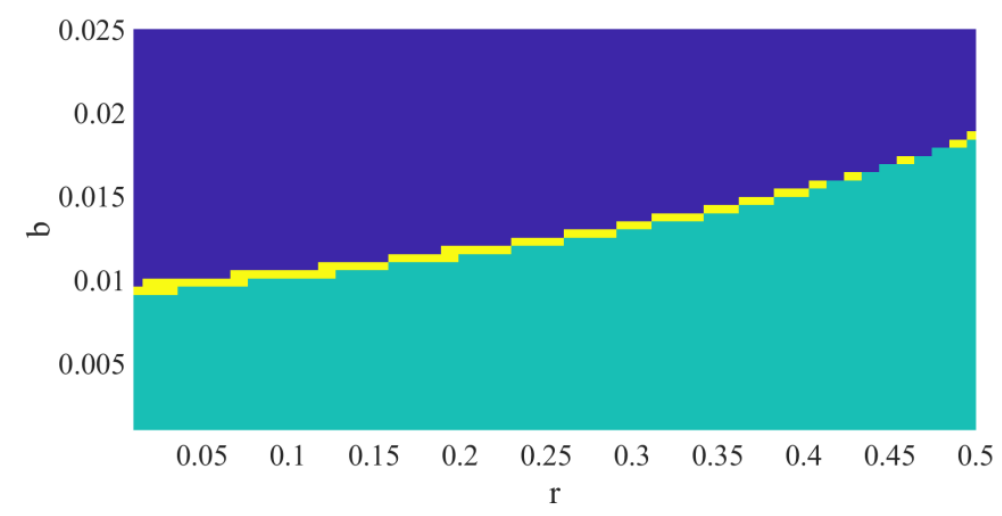

Figure 4: Stability results

Nevertheless, some conservatism remains in the solution that can also be dropped with the use of static multipliers as in (Heath and Li, 2010), but the proof of the existence of such multipliers will be presented in a forthcoming publication. A simulation is carried out for $r=0.2$ and the system remains stable for a set-point tracking. The model reduction is efficient which means that the actual upper bound of the error is below 0.01 . This $b$ and $r$ correspond to a stable region as predicted above. Figure 5 shows the output temperature for different set-points while Figure 6 shows the corresponding cooling temperatures.

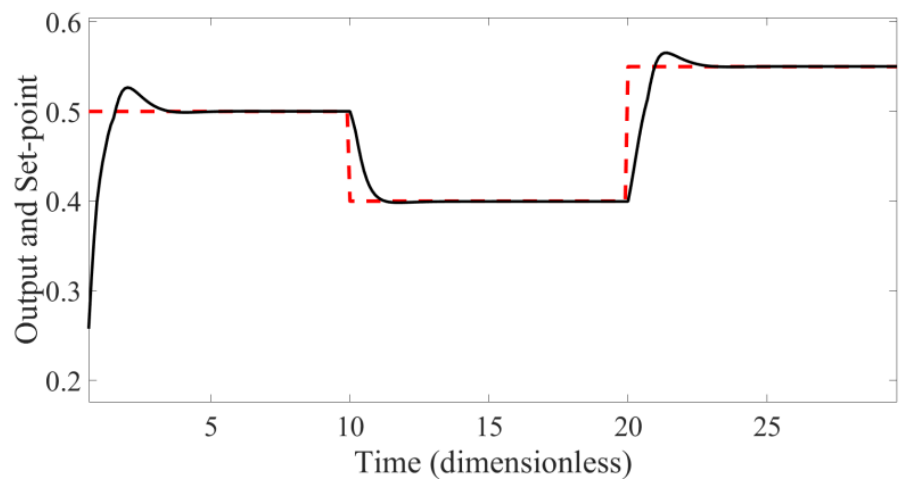

Figure 5: Output and set-points against time 


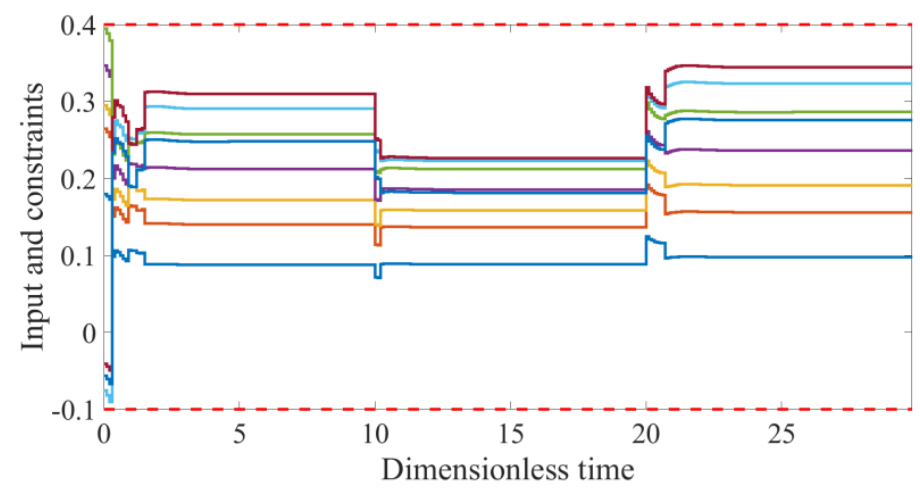

Figure 6: Cooling temperature against time

The semi-definite programming problems are all solved using MATLAB r2017a with YALMIP (Löfberg, 2004) and MOSEK (ApS, 2017) in machine with Intel r 17-6700@3.40GHz and 16.0 GB of RAM

\section{Conclusion}

This paper considers the global stability for processes that have been modelled with a piecewise affine model under unstructured uncertainty, following the analyses proposed in (Heath et al., 2006) and (Petsagkourakis et al., 2018). Linearization and model reduction reduce the complexity of the nonlinear PDE model but create uncertainties. We construct necessary conditions for PWA models when relaxed barrier-based MPC is employed under general nonlinear convex constraints. These conditions are developed through the use of conic combination for the integral quadratic constraints taking advantage of the excess positivity that the nonlinearity may have. Common and PWQ storage functions have been employed and for the case study presented that the use of PWQ was not needed as it produced the same results with a common storage function with conic combination. This methodology can be used for efficient global stability analysis of dissipative nonlinear closed-loop systems. In the future, more generic multipliers should be considered as in (Heath and $\mathrm{Li}, 2010$ ) as well as the inclusion of state constraints, taking advantage of the relaxed barrier. In addition, if additional information for the uncertainty is available, then performance criteria can be incorporated as in the synthesis of anti-windup schemes (Adegbege and Heath, 2017).

\section{Acknowledgements}

The authors wish to thank the University of Manchester for the provision of a President's Doctoral Scholarship Award to Panagiotis Petsagkourakis

\section{References}

ApS, M., 2017. The MOSEK optimization toolbox for MATLAB manual. Version 8.

Armaou, A., Kevrekidis, I.G., Theodoropoulos, C., 2005. Equation-free gaptooth-based controller design for distributed complex/multiscale processes. Comput. Chem. Eng. 29, 731-740.

Bemporad, A., Morari, M., Dua, V., Pistikopoulos, E.N., 2002. The explicit linear quadratic regulator for constrained systems. Automatica 38, 3-20. 
Bock, H.G., Diehl, M., Kühl, P., Kostina, E., Schiöder, J.P., Wirsching, L., 2007. Numerical Methods for Efficient and Fast Nonlinear Model Predictive Control, in: Findeisen, R., Allgöwer, F., Biegler, L.T. (Eds.), Assessment and Future Directions of Nonlinear Model Predictive Control. Springer Berlin Heidelberg, Berlin, Heidelberg, pp. 163-179.

Bonis, I., Theodoropoulos, C., 2012. Model reduction-based optimization using large-scale steady-state simulators. Chem. Eng. Sci. 69, 69-80.

Bonis, I., Xie, W., Theodoropoulos, C., 2014a. Multiple model predictive control of dissipative PDE systems. IEEE Trans. Control Syst. Technol. 22, 1206-1214.

Bonis, I., Xie, W., Theodoropoulos, C., 2014b. Multiple model predictive control of dissipative PDE systems. IEEE Trans. Control Syst. Technol. 22, 1206-1214.

Bonis, I., Xie, W., Theodoropoulos, C., 2012. A Linear Model Predictive Control Algorithm for Nonlinear Large-Scale Distributed Parameter Systems. AIChE JournalAIChE J. 58, 801-811.

Boyd, S., Vandenberghe, L., 2004. Convex Optimization. Cambridge University Press, New York, NY, USA.

Breschi, V., Piga, D., Bemporad, A., 2016. Piecewise affine regression via recursive multiple least squares and multicategory discrimination. Automatica 73, 155-162.

Christofides, P.D., 2012. Nonlinear and Robust Control of PDE Systems: Methods and Applications to Transport-Reaction Processes, Systems \& Control: Foundations \& Applications. Birkhäuser Boston.

Du, J., Song, C., Yao, Y., Li, P., 2013. Multilinear model decomposition of MIMO nonlinear systems and its implication for multilinear model-based control. J. Process Control 23, 271-281.

Feller, C., Ebenbauer, C., 2017. A stabilizing iteration scheme for model predictive control based on relaxed barrier functions. Automatica 80, 328-339.

Feller, C., Ebenbauer, C., 2016. Robust stability properties of MPC iteration schemes based on relaxed barrier functions 1484-1489.

Feller, C., Ebenbauer, C., 2015a. Relaxed Logarithmic Barrier Function Based Model Predictive Control of Linear Systems.

Feller, C., Ebenbauer, C., 2015b. Weight recentered barrier functions and smooth polytopic terminal set formulations for linear model predictive control. Proc. Am. Control Conf. 2015-July, 1647-1652.

Hastie, T., Tibshirani, R., Friedman, J., 2009. The Elements of Statistical learning: Data Miningm Inference and Prediction.

Heath, W.P., Li, G., 2010. Multipliers for model predictive control with structured input constraints. Automatica $46,562-568$.

Heath, W.P., Li, G., Wills, A.G., Lennox, B., 2006. The robstness of input constrained model predictive control to infinity-norm model uncertainty. IFAC Proc. Vol. 39, 495-500.

Heath, W.P., Wills, A.G., Akkermans, J.A.G., 2005. A sufficient condition for the stability of optimizing controllers with saturating actuators. Int. J. Robust Nonlinear Control 15, 515-529. 
Hovland, S., Lovaas, C., Gravdahl, J.T., Goodwin, G.C., 2008. Stability of Model Predictive Control Based on Reduced-Order Models. Decis. Control. 2008. CDC 2008. 47th IEEE Conf. 4067-4072.

Jerez, J.L., Goulart, P.J., Richter, S., Constantinides, G. a, Kerrigan, E.C., Morari, M., 2014. Embedded Online Optimization for Model Predictive Control at Megahertz Rates. Autom. Control. IEEE Trans. 59, 32383251 .

Jönsson, U., Rantzer, A., 2000. 6. Optimization of Integral Quadratic Constraints, in: Advances in Linear Matrix Inequality Methods in Control. pp. 109-127.

Löfberg, J., 2004. YALMIP : A Toolbox for Modeling and Optimization in MATLAB, in: In Proceedings of the CACSD Conference. Taipei, Taiwan.

Løvaas, C., Seron, M.M., Goodwin, G.C., 2010. Robust output-feedback MPC with integral action. IEEE Trans. Automat. Contr. 55, 1531-1543.

Lozano, R., Maschke, B., Brogliato, B., Egeland, O., 2000. Dissipative Systems Analysis and Control: Theory and Applications. Springer-Verlag, Berlin, Heidelberg.

Mayne, D.Q., 2014. Model predictive control: Recent developments and future promise. Automatica 50, 29672986.

Megretski, A., Rantzer, A., 1997. System Analysis via Integral Quadratic Constraints Part II System Analysis via Integral Quadratic Constraints Part IIa : Abstract theory. Control 42, 819-830.

Narasingam, A., Kwon, J.S.-I., 2017. Development of local dynamic mode decomposition with control: Application to model predictive control of hydraulic fracturing. Comput. Chem. Eng. 106, 501-511.

Patrinos, P., Bemporad, A., 2013. Proximal Newton methods for convex composite optimization, in: 52nd IEEE Conference on Decision and Control. pp. 2358-2363.

Petsagkourakis, P., Heath, W.P., Theodoropoulos, C., 2018. Stability Analysis of Piecewise Affine Systems with Multi-model Model Predictive Control. arXiv:1808.00307.

Pfifer, H., Seiler, P., 2016. Robustness analysis of linear parameter varying systems using integral quadratic constraints. Int. J. Robust Nonlinear Control 18, 557-569.

Pfifer, H., Seiler, P., 2015. An Overview of Integral Quadratic Constraints for Delayed Nonlinear and Parameter-Varying Systems 1-15.

Rantzer, A., 2012. On the Kalman-Yakubovich-Popov Lemma for Positive Systems 7482-7484.

Rantzer, A., Johansson, M., 2000. Piecewise linear quadratic optimal control. IEEE Trans. Automat. Contr. 45, 629-637.

Rewienski, M., White, J., 2003. A trajectory piecewise-linear approach to model order reduction and fast simulation of nonlinear circuits and micromachined devices. IEEE Trans. Comput. Des. Integr. Circuits Syst. 22, 155-170.

Shroff, G.M., Keller, H.B., 1993. Stabilization of Unstable Procedures: The Recursive Projection Method. SIAM J. Numer. Anal. 30, 1099-1120. 
Sopasakis, P., Bernardini, D., Bemporad, A., 2013. Constrained Model Predictive Control Based on ReducedOrder Models 7071-7076.

Stella, L., Themelis, A., Sopasakis, P., Patrinos, P., 2017. A Simple and Efficient Algorithm for Nonlinear Model Predictive Control.

Temam, R., 1997. Infinite-Dimensional Dynamical Systems in Mechanics and Physics, Applied Mathematical Sciences. Springer New York.

Theodoropoulos, C., 2011. Optimisation and linear control of large scale nonlinear systems: A review and a suite of model reduction-based techniques, in: Lecture Notes in Computational Science and Engineering Vol 75. pp. 37-61.

Theodoropoulos, C., Qian, Y.-H., Kevrekidis, I.G., 2000. "Coarse” stability and bifurcation analysis using timesteppers: A reaction-diffusion example. Proc. Natl. Acad. Sci. 97, 9840-9843.

Wang, Y., Boyd, S., 2010. Fast Model Predictive Control Using Online Optimization. Control Syst. Technol. IEEE Trans. 18, 267-278.

Wills, A.G., Heath, W.P., 2004. Barrier function based model predictive control. Automatica 40, 1415-1422.

Wills, A.G., Heath, W.P., 2004. Barrier function based model predictive control. Automatica 40, 1415-1422.

Yakubovich, V.A., 1967. Frequency conditions for the absolute stability of control systems with several nonlinear or linear nonstationary blocks. Avtomat. i Telemekh. 5-30.

Zelik, S., 2014. Inertial manifolds and finite-dimensional reduction for dissipative PDEs. Proc. R. Soc. Edinburgh Sect. A Math. 144, 1245-1327. 